\title{
Assessment of pulmonary toxicities in breast cancer patients undergoing treatment with anthracycline and taxane based chemotherapy and radiotherapy- a prospective study
}

\author{
Aramita Saha ${ }^{1}$, Subrata Chattopadhyay ${ }^{2}$ \\ ${ }^{I}$ Department of Medical Oncology, Apollo Gleangles Cancer Hospital, Kolkata, India. \\ ${ }^{2}$ Department of Radiation Oncology, Medical College, Kolkata, India.
}

Received October 02, 2013; Revised October 27, 2013; Accepted October 27, 2013; Published Online November 22, 2013

\section{Original Article}

\begin{abstract}
Background: Anthracycline based regiments and/or taxanes and adjuvant radiotherapy; the main modalities of treatment for breast cancers are associated with deterioration of pulmonary functions and progressive pulmonary toxicities. Aim: Assessment of pulmonary toxicities and impact on pulmonary functions mainly in terms of decline of forced vital capacity (FVC) and the ratio of forced expiratory volume (FEV) in 1 Second and FEV1/FVC ratio with different treatment times and follow ups in carcinoma breast patients receiving anthracycline and/or taxane based chemotherapy and radiotherapy. Materials and methods: A prospective single institutional cohort study was performed with 58 breast cancer patients between January 2011 to July 2012 who received either anthracycline based (37 patients received 6 cycles FAC $=5$ FU, Adriamycin, Cyclophosphamide regime) and radiotherapy or anthracycline and taxane based chemotherapy (21 patients received 4cycles AC= Adriamycin, Cyclophosphamide; followed by 4 cycles of $\mathrm{T}$ =Taxane) and radiotherapy. Assessment of pulmonary symptoms and signs, chest $\mathrm{x}$-ray and pulmonary function tests were performed at baseline, midcycle, at end of chemotherapy, at end radiotherapy, at 1 and 6 months follow ups and compared. By means of a two-way analysis of variance (ANOVA) model, the course of lung parameters across the time points was compared. Results and Conclusion: Analysis of mean forced vital capacities at different points of study times showed definitive declining pattern, which is at statistically significant level at the end of $6^{\text {th }}$ month of follow up ( $\mathrm{p}=0.032$ ) .The FEV1/FVC ratio (in percentage) also revealed a definite decreasing pattern over different treatment times and at statistically significant level at $6^{\text {th }}$ month follow up with p value 0.003 . Separate analysis of mean FEV1/FVC ratios over time in anthracycline based chemotherapy and radiotherapy group as well as anthracycline and taxane based chemotherapy and radiotherapy group showed a similar declining pattern.
\end{abstract}

Keywords: Anthracycline, Taxane, Radiotherapy, Pulmonary, FEV1/FVC

\section{Introduction}

Breast cancer is the leading cause of cancer death among women around the world. ${ }^{1}$ It accounts for $26 \%$ of all malignancies in women and second most common cause of cancer death in women. ${ }^{2}$ In India it shows mixed incidence pattern with breast cancer being second to cancer of the cervix in

Corresponding author: Aramita Saha; Department of Medical Oncology, Gleangles Cancer Hospital, Kolkata, India.

Email: docaramita@gmail.com

Cite this article as:

Saha A, Chattopadhyay S. Assessment of pulmonary toxicities in breast cancer patients undergoing treatment with anthracycline and taxane based chemotherapy and radiotherapy- a prospective study. Int J Cancer Ther Oncol 2013; 1(2):01021.

DOI: 10.14319/ijcto.0102.1 rural areas ${ }^{1,2,}$; however, in metropolitan cities the incidence of breast cancer has crossed that of cervix. ${ }^{2}$ Mathematical models suggest that both the adoption of screening mammography and the availability of adjuvant chemotherapy, radiotherapy and tamoxifen have contributed approximately equally to the improvement of breast cancer outcomes. ${ }^{3}$

Adjuvant chemotherapy reduces local recurrence after radiation therapy in breast cancer. Anthracycline (doxorubicin)-based regimens ( \pm taxanes for high-risk disease) have been associated with superior outcomes as compared to nonanthracycline containing regimens. Recent evidence suggests that disease free survival (DFS) and overall survival (OS) of breast cancers has been increased with taxane-based therapy as compared to anthracycline-based therapy. Neoadjuvant chemotherapy is considered standard of care in 
high-risk populations such as young patients and/or advanced stage disease, and has been evaluated in Stage II-IIIa breast cancer in randomized trials. ${ }^{3,4}$

Paclitaxel (Taxol) is one of the most potent chemotherapeutic agents in the treatment of breast cancers has experienced widespread use over the past 5 years. . A limited number of case reports are available concluding that Taxol is associated with respiratory symptoms, including dyspnea, cough, wheezing and chest tightness..$^{5}$ Interstitial and reticulonodular infiltrates have been described on chest radiographic examination in few studies with paclitaxel in breast cancers. ${ }^{6}$ Cases of transient pulmonary infiltrates and suspected interstitial pneumonitis have been reported, although the true incidence of lung toxicity that is directly related to paclitaxel is not well understood. A prospective study of lung function in 33 patients who received paclitaxel with carboplatin (an agent with little evidence for direct lung toxicity) for nonthoracic malignancy revealed an isolated decrease in diffusing capacity without other clinical or radiographic evidence of pulmonary toxicity.$^{7}$ Clinicians should be aware of the potential for paclitaxel to impair pulmonary function.

Randomized trials have found that addition of adjuvant therapy with taxane to an anthracycline-based chemotherapy regimen, compared with anthracycline-based chemotherapy alone, led to improved survival for high grade breast cancer patients. ${ }^{8,9}$ Irradiation is also an important adjuvant therapy for breast cancer. Specifically, adjuvant radiation therapy for selected patients with breast cancer reduces locoregional recurrence and improves overall survival. ${ }^{10,11}$

One serious potential risk of radiation therapy for breast cancer is symptomatic radiation pneumonitis. Fortunately, with modern irradiation techniques, the risk of radiation pneumonitis is low (5\%), and its course is usually self-limited .${ }^{12}$ However, the risk of radiation pneumonitis has recently become a greater clinical concern because of reports suggesting that this risk may increase in patients who receive taxanes.

It is well known that post-operative adjuvant loco-regional radiotherapy in breast cancer is associated with pulmonary complications. The frequency and grade of pulmonary complications following radiotherapy for breast cancer are, however, still debated. Few investigators have quantified this problem by using objective methods such as pulmonary function tests (PFTs). ${ }^{13}, 14$ The PFTs have the advantage of being widely available and reproducible methods for detecting parenchymal lung damage, if they are performed under strict standardized conditions. ${ }^{15} \mathrm{We}$ performed a prospective study from January 2011 to August 2012 for pulmonary status and pulmonary toxicities assessment on 58 Breast cancer patients attended and registered Radiotherapy department of Medical College, Kolkata, who received either anthracycline based and/or taxane based chemotherapy followed by radiotherapy. The effects of anthracycline and taxane based chemotherapy and radiotherapy on pulmonary functions were assessed and variation of pulmonary toxicities with different treatment times and follow up were analysed.

\section{Methods and Materials}

The lung is one of the major dose-limiting organs for radiotherapy within the thorax. Therefore, the total dose that can safely be delivered to patients with malignant tumors like carcinoma breast has to be limited because of the risk of radiation pneumonitis (developing 1 to 6 months after treatment) and radiation fibrosis (developing from 6 months onward). Chemotherapy (regardless of the type of drug) primarily affected the diffusion capacity. So the aim of our study was to analyze whether and how much the course in pulmonary function changes over time, forced vital capacity [FVC], ratio of forced expiratory volume in 1second [FEV1/FVC ratio] varied between the different treatment regimens and whether recovery of early pulmonary damage occurred or not. In general, when chemotherapy and radiotherapy are combined, two different effects may occur as an interaction between both modalities, resulting in an enhancement of radiation-induced damage or an additional effect.

Study Area: Department of Radiotherapy, Medical College and Hospitals, Kolkata.

Study Population: All biopsy proven cases of left sided female carcinoma breast attending the Radiotherapy Out $\mathrm{Pa}$ tients' Department and conforming to inclusion /exclusion criteria mentioned herein.

Study Period: Case accrual started from January 2011 and it was divided into preparatory phase, data collection phase data compilation phase, data analysis phase and preparation phase.

\section{Sample Size: 60 patients}

Sample Design: All the population with carcinoma breast (female) who conform to the inclusion / exclusion criteria mentioned herein and gave consent to be included in the study.

\section{Inclusion Criteria:}

- Female carcinoma breast patients.

- $\quad$ Age above 20 years and below 70 years.

- Those who are eligible to receive anthracycline and/or taxane based chemotherapy and external beam radiotherapy.

- $\quad$ Stage II and onwards (American Joint Committee on Cancer Stage Grouping).

- $\quad$ Karnofsky performance status $>70$. 
- Baseline normal hematological, renal and hepatic profile.

- Baseline normal cardiac and pulmonary function status.

- Informed Consent to participate in this study.

\section{Exclusion Criteria:}

- Male carcinoma breast patients.

- $\quad$ Patients undergoing breast conservation surgery.

- Pregnant and lactating patients.

- Any evidence of Metastasis at presentation.

- $\quad$ Previous history of any malignancy.

- Previously received / currently receiving chemotherapy / radiotherapy.

- $\quad$ Not enrolled in any other study on Breast cancer.

Study Design: Prospective observational single-Institutional trial.

\section{Parameters studied}

- History.

- Detailed physical examination.

- Pulmonary function test (FEV1, FVC, FEV1/FVC ratio and $\mathrm{VC})$

- $\quad$ Chest X Ray (PA and lateral view).

Study Techniques: All biopsy proven cases of left sided carcinoma breast attending the OPD of Radiotherapy, Medical College Hospitals, Kolkata, satisfying the specified Inclusion and Exclusion Criteria who are willing to participate in the study were included.

Patients were treated with Anthracycline and / or Taxane based chemotherapy in the Neo-Adjuvant or Adjuvant setting in combination with Modified Radical Mastectomy (Timing of surgery depending on operability). In addition all patients will receive adjuvant External Beam Radiotherapy (Dose, Portals etc depending on stage and tumour features). Hormone receptor positive patients will receive adjuvant Endocrine therapy for a minimum duration of five years.

All patients were subjected to chest $\mathrm{X}$-ray and Pulmonary function tests (Clinical assessment, Chest $\mathrm{X}$ ray, FEV1, FVC, FEV1/FVC) at baseline, during and at the end of chemotherapy, at the end of radiation and during first month and sixth month follow up.Mid cycle chemotherapy means after completion of 3rd cycle of chemotherapy in FAC group and after completion of 4th cycle of chemotherapy in Anthracycline, Cyclophosphamide (AC) followed by Taxane (T group).

\section{Statistical analysis:}

Quantitative variables were compared between two groups using an unpaired t test for normally distributed variables or Wilcoxon two sample tests for skewed distributed variables.
Normally distributed variables are reported as mean, standard deviation, and variance. Skewed distributed variables are reported as median and range (minimum to maximum).

By means of a two-way ANOVA model, the course of lung parameters (decline of pulmonary function tests) across the time points was compared between patients. Correlations between variables were calculated using Pearson's or Spearman correlation coefficient test as appropriate. All $\mathrm{P}$ values were two-sided and $\mathrm{P}<0.05$ was considered statistically significant. Medcalc and Vassarstats were used to perform the statistical analysis.

\section{Results}

\section{Case Accrual}

Initially, 60 patients were selected for accrual. Of these, after careful scrutiny towards meeting of inclusion and exclusion criteria, 58 breast cancer patients were actually found suitable. All patients under study had left sided breast cancer. However, 4 patients were expired during and at the end of chemotherapy and were consequently excluded from the study. Ultimately 54 patients underwent the study till end.

\section{Baseline Patient Characteristics}

\begin{tabular}{ccc}
\multicolumn{2}{c}{ TABLE 1: Baseline age distribution of the study population } \\
\hline \hline Age Groups & No of Patients & Percentage (\%) \\
\hline 20-32 years & 9 & 15.52 \\
33-45 years & 18 & 31.03 \\
46-58 years & 23 & 39.65 \\
$>58$ years & 8 & 13.79 \\
\hline \hline
\end{tabular}

The baseline age distributions of patients under are depicted in Table 1. The Mean age is 51.5 yrs. Inclusion criteria of our study was lump size more than $5 \mathrm{~cm}$ and / or node positive disease. All N0 patients must have T3 (tumour $>5 \mathrm{~cm}$ ) according to our study inclusion criteria otherwise node positive.

All the patients in the study population received anthracycline and/or taxane based chemotherapy and post chemotherapy radiation of chest flap alone or in combination with supraclavicular field radiotherapy or chest flap+supraclavicular field +axillary field radiation. All patients have undergone mastectomy as the very first treatment or initially received 2-3 cycles of neoadjuvant chemotherapy, downstaged and then undergone mastectomy; after that completed chemotherapy. After completion of chemotherapy they received radiation .At the end of chemotherapy the study population came down to 54.Two patients died after 4 cycles, 1 patient after 5 th cycle of chemotherapy and 1 patient after 2 nd cycle of chemotherapy. So, 54 patients have undergone radiotherapy.

Among the 58 patients, 37 patients (63.79\%) received only 
anthracycline based (doxorubicin) chemotherapy and 21 patients $(36.2 \%)$ received both anthracycline and taxane both based chemotherapy. Moreover, majority of the patients belonged to poor socio-economic status; received doxorubicin, which is supplied free of cost from government fund in our ward. So we have chosen doxorubicin not epirubicin as anthracycline regime. For those 37 patients who received only anthracycline based chemotherapy, the regime chosen was 6cycles of FAC every 21days(Inj 5FU 500mg/m2 iv D1;Inj Doxorubicin 50mg/m2 iv D1; Inj Cyclophosphamide $500 \mathrm{mg} / \mathrm{m} 2$ iv D1). Rest 21 patients received both anthracycline and taxane based chemotherapy with 4 cycles of AC followed by 4 cycles of T(Inj Doxorubicin 60mg/m2 iv D1,Inj Cyclophosphamide $600 \mathrm{mg} / \mathrm{m} 2$ iv D1 every 21 days 4 cycles followed by Paclitaxel $175 \mathrm{mg} / \mathrm{m} 2$ iv D1 every 21days 4 cycles. Among 58 patients, 42 patients received neoadjuvant chemotherapy 2-3 cycles, thereafter achieved complete or partial response, undergone MRM and axillary clearance, thereafter completed remaining cycles of chemotherapy. Remaining 16 patients have undergone MRM with axillary clearance first, thereafter received adjuvant chemotherapy.54 patients have undergone radiotherapy after completion of chemotherapy.

\section{Evaluation of pulmonary toxicities following chemo- therapy and radiotherapy in breast cancer patients}

The baseline pulmonary function tests and chest $\mathrm{x}$-ray including clinical features were within normal limits in all patients of the study population.

Assessment of respiratory symptoms: Cough and respiratory distress were considered as respiratory symptoms.

a) Respiratory distress: Total seven patients, three in the anthracycline based CT+RTgroup (one during third cycle of chemotherapy, two during first cycle of chemotherapy) complained of respiratory distress. Two patients in the anthracycline and taxane based $\mathrm{CT}+\mathrm{RT}$ group developed respiratory distress during first cycle of chemotherapy; two patients during radiotherapy. 1 patient during $1 \mathrm{month}$ follow up and 3 patients in 6th month of follow up complained of moderate respiratory distress. From symptoms only we couldn't distinguish that whether the distress was due to cardiological or pulmonary damage .Patients were urgently sent for immediate cardiological evaluation -and chest $\mathrm{x}$-ray. None revealed any significant cardiological abnormality. The patient who developed respiratory distress during 1 month follow up revealed bilateral lung metastasis in chest $\mathrm{x}$-ray and among those 3 who developed distress at 6th month follow up; 1 revealed lung metastasis and 2 showed overt features of radiation pneumonitis in chest $\mathrm{x}$-ray. So, only 2 patients $(3.44 \%)$ of the entire study group revealed radiation pneumonitis at 6th month of follow up. b) Cough: Total 9 patients in the entire study group developed cough. Two during $3^{\text {rd }}$ cycle chemotherapy, three during 1 month follow up and four during 6th month of follow ups. Investigations revealed lung metastasis in total 2 patients and 2 patients developed radiation pneumonitis.

Evaluation of respiratory signs: None of the patients revealed any overt respiratory signs.

Evaluation of pulmonary toxicities by study tools: All the patients have undergone chest $\mathrm{x}$ ray and pulmonary function tests(FVC and FEV1/FVC ratio) at baseline, at mid cycle chemotherapy, at the end of chemotherapy,at completion of radiotherapy, at 1 moth and 6 th month of follow ups.

Interpretation of chest $\mathbf{x}$-ray: Two patients in the entire study group revealed features of lung metastases in chest $\mathrm{x}$-ray, one at 1 st month of follow up and one at 6th month of follow up. Only 2 patients (3.44\%) in the entire study group developed features of radiation pneumonitis on chest $\mathrm{x}$-ray at 6th month of follow up. Both the patients received anthracycline and taxane based chemotherapy and radiotherapy.

\section{Interpretation of pulmonary function tests:}

TABLE 2: Variation of mean FEV1/FVC ratios (\%) at different points of time and follow ups.

\begin{tabular}{|c|c|c|c|c|c|}
\hline & Mean & Median & SD & $\begin{array}{l}\text { Variance } \\
(\mathrm{SD})\end{array}$ & $\begin{array}{l}\text { Population } \\
\text { (SD) }\end{array}$ \\
\hline Baseline & 79.17 & 78.9 & 9.24 & 10.05 & 1.14 \\
\hline $\begin{array}{c}\text { FEV1/FVC } \\
(\mathrm{N}=58)\end{array}$ & $(0.791)$ & $(0.789)$ & & & \\
\hline Mid cycle CT & 78.19 & 77.9 & 6.87 & 5.82 & 1.38 \\
\hline $\begin{array}{c}\text { FEV1/FVC } \\
(\mathrm{N}=58)\end{array}$ & $(0.781)$ & $(0.779)$ & & & \\
\hline End CT & 76.15 & 75.2 & 1.94 & 3.78 & 1.96 \\
\hline $\begin{array}{l}\text { FEV1/FVC } \\
\quad \mathrm{N}=54)\end{array}$ & $(0.761)$ & $(0.752)$ & & & \\
\hline End RT & 74.24 & 74.56 & 2.32 & 5.4 & 2.3 \\
\hline $\begin{array}{c}\text { FEV1/FVC } \\
(\mathrm{N}=54)\end{array}$ & $(0.742)$ & $(0.745)$ & & & \\
\hline 1 month FU & 72.59 & 72.2 & 2.45 & 6.7 & 2.42 \\
\hline $\begin{array}{c}\text { FEV1/FVC } \\
(\mathrm{N}=52)\end{array}$ & $(0.725)$ & $(0.722)$ & & & \\
\hline $\begin{array}{c}6 \text { months FU } \\
\text { FEV1/FVC } \\
(\mathrm{N}=50)\end{array}$ & $\begin{array}{c}69.52 \\
(0.695)\end{array}$ & $\begin{array}{l}69.075 \\
(0.69)\end{array}$ & 2.74 & 74 & 7.20 \\
\hline
\end{tabular}

Analysis of mean forced vital capacities at different points of study time (baseline, mid cycle chemotherapy, end of chemotherapy, end of radiotherapy, 1 month and 6 months follow ups showed definitive declining pattern, which is at statistically significant level at the end of 6 th month of follow up $(\mathrm{p}=0.032)$ in the entire study population (Table 2 , Table 3 , and Figure 1). 
TABLE 3: Variation of means of FVCs (forced vitals capacities in liters) over different treatment times:

\begin{tabular}{ccc}
\hline \hline & Mean FVCs & P-value \\
\hline Baseline & 2.84 & -- \\
MID CT & 2.69 & 0.99 \\
END CT & 2.54 & 0.867 \\
END RT & 2.19 & 0.740 \\
1 month follow up & 2.06 & 0.632 \\
6 month follow up & 1.81 & 0.032 \\
\hline \hline
\end{tabular}

$\mathrm{P}$ values were compared to baseline; $\mathrm{P}<0.05$ "means statistically significant"

TABLE 4: Comparison of mean FEV1/FVC ratios with baseline at different times of study time

\begin{tabular}{cc}
\hline \hline Different points of study time & P-value \\
\hline Baseline and mid cycle chemotherapy & 0.98 \\
Baseline and end of chemotherapy & 0.76 \\
Baseline and end of radiotherapy & 0.71 \\
Baseline and 1 month follow up & 0.068 \\
Baseline and 6 months follow up & 0.003 \\
\hline \hline
\end{tabular}

$\mathrm{P}$ values of mean FEV1/FVC ratio as compared to baseline; $\mathrm{P}<0.05$ "means statistically significant"

TABLE 5: Variation of mean FEV1/FVC ratios (\%) s at different points of time and follow up period in anthracycline based chemotherapy and radiotherapy group

\begin{tabular}{cccccc}
\hline \hline & Mean & Median & SD & $\begin{array}{c}\text { Variance } \\
(\text { SD })\end{array}$ & $\begin{array}{c}\text { Population } \\
(\mathrm{SD})\end{array}$ \\
\hline $\begin{array}{c}\text { Baseline FEV1/FVC } \\
(\mathrm{N}=37)\end{array}$ & $79.82(0.798)$ & $79.2(0.792)$ & 1.32 & 1.75 & 1.3 \\
$\begin{array}{c}\text { Mid cycle CT FEV1/FVC } \\
(\mathrm{N}=37)\end{array}$ & $78.36(0.736)$ & $77.8(0.778)$ & 1.97 & 2.1 & 1.48 \\
$\begin{array}{c}\text { End CT FEV1/FVC } \\
(\mathrm{N}=34)\end{array}$ & $76.59(0.765)$ & $76.5(0.765)$ & 2.06 & 4.02 & 1.97 \\
$\begin{array}{c}\text { End RT FEV1/FVC } \\
(\mathrm{N}=34)\end{array}$ & $75.05(0.75)$ & $75.45(0.754)$ & 2.23 & 5.01 & 2.02 \\
$\begin{array}{c}1 \text { month FU FEV1/FVC } \\
(\mathrm{N}=33)\end{array}$ & $73.45(0.734)$ & $73.5(0.735)$ & 2.4 & 5.77 & 2.36 \\
$6 \begin{array}{c}6 \text { months FU FEV1/FVC } \\
(\mathrm{N}=31)\end{array}$ & $69.9(0.699)$ & $69.5(0.695)$ & 3.2 & 10.24 & 3.14 \\
\hline \hline
\end{tabular}

Abbreviations: CT = Chemotherapy; FU = Follow up

TABLE 6: Variation of mean FEV1/FVC ratios with baseline at different times of study time of the study group received anthracycline+ taxane

\begin{tabular}{|c|c|c|c|c|c|}
\hline & Mean & Median & SD & $\begin{array}{l}\text { Variance } \\
\text { (SD) }\end{array}$ & $\begin{array}{l}\text { Population } \\
\text { (SD) }\end{array}$ \\
\hline $\begin{array}{l}\text { Baseline FEV1/FVC } \\
(\mathrm{N}=21)\end{array}$ & $\begin{array}{c}78.92 \\
(0.789)\end{array}$ & $\begin{array}{c}78.7 \\
(0.787)\end{array}$ & 3.46 & 8.48 & 6.45 \\
\hline $\begin{array}{l}\text { Mid cycle CT FEV1/FVC } \\
\qquad(\mathrm{N}=21)\end{array}$ & $\begin{array}{c}77.86 \\
(0.778)\end{array}$ & $\begin{array}{c}77.6 \\
(\mathrm{O} .776)\end{array}$ & 1.93 & 6.7 & 5.98 \\
\hline $\begin{array}{c}\text { End CT FEV1/FVC } \\
(\mathrm{N}=20)\end{array}$ & $\begin{array}{c}75.4 \\
(0.754)\end{array}$ & $\begin{array}{c}75.2 \\
(0.752)\end{array}$ & 1.61 & 2.61 & 1.57 \\
\hline $\begin{array}{c}\text { End RT FEV1/FVC } \\
(\mathrm{N}=20)\end{array}$ & $\begin{array}{c}72.85 \\
(0.728)\end{array}$ & $\begin{array}{c}72.5 \\
(0.725)\end{array}$ & 1.78 & 3.17 & 1.73 \\
\hline $\begin{array}{c}1 \text { month FU FEV1/FVC } \\
(\mathrm{N}=19)\end{array}$ & $\begin{array}{c}71.09 \\
(0.719)\end{array}$ & $\begin{array}{l}72.01 \\
(0.721)\end{array}$ & 1.74 & 3.03 & 1.69 \\
\hline $\begin{array}{c}6 \text { months FU } \\
\text { FEV1/FVC }(\mathrm{N}=19)\end{array}$ & $\begin{array}{c}68.79 \\
(0.687)\end{array}$ & $\begin{array}{c}68.45 \\
(0.684)\end{array}$ & 1.6 & 2.56 & 1.55 \\
\hline
\end{tabular}

Abbreviations: CT = Chemotherapy; FU = Follow up; RT = Radiation therapy 


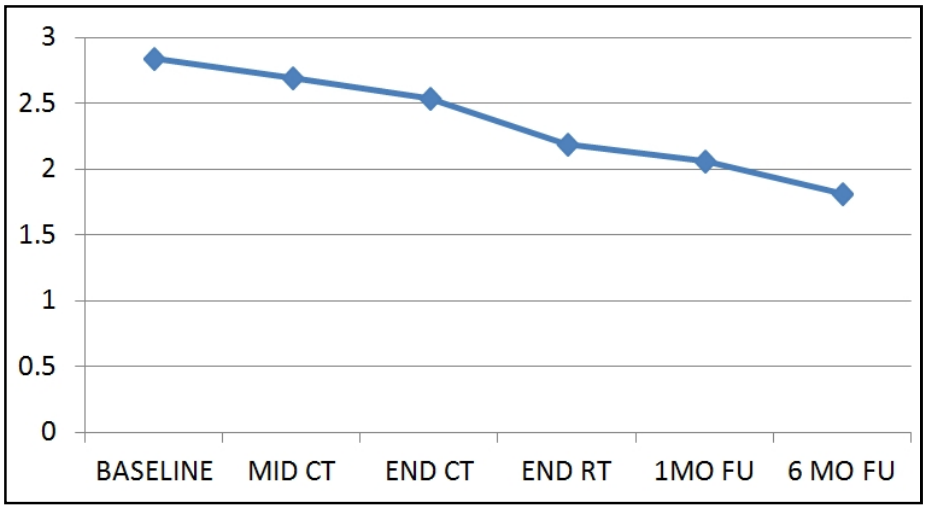

FIG. 1: Graphical representation of variations of mean forced vital capacities (FVC) over different treatment times and follow ups $\mathrm{Y}$-axis represents Mean FVC s over time

Abbreviations: $\mathrm{CT}=$ Chemotherapy, $\mathrm{RT}=$ Radiotherapy, FU= Follow up, FAC=5 Fluorouracil, Adriamycin, Cyclophosthamide, $\mathrm{AC}=\mathrm{Adriamycin}$, Cyclophosphamide, $\mathrm{T}=$ Taxane

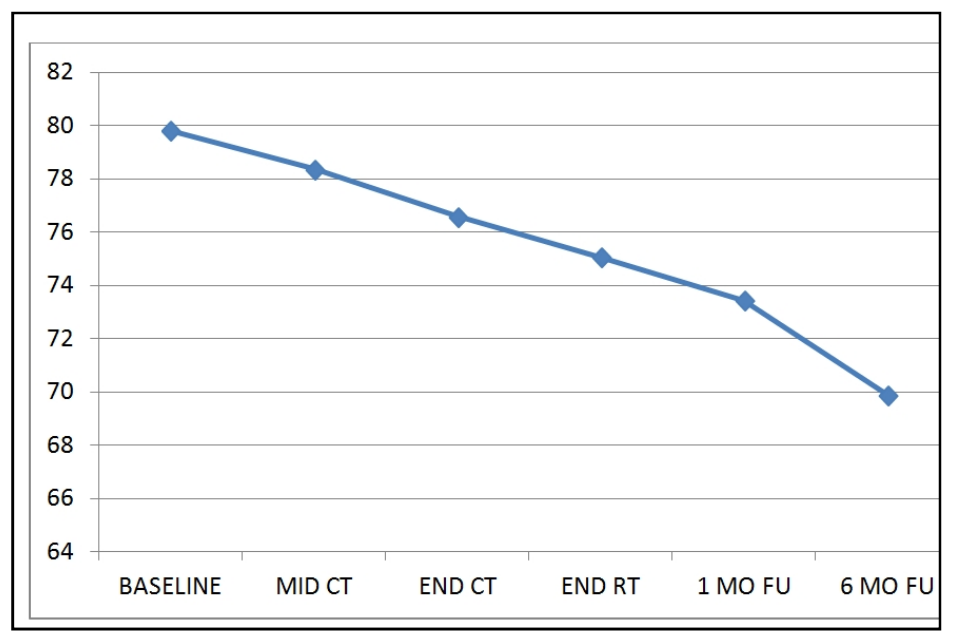

FIG. 2: Graphical representation of pattern of decline of FEV1/FVC ratio in the entire study group. $\mathrm{Y}$-axis represents FEV1/FVC ratios

Abbreviations: $\mathrm{CT}=$ Chemotherapy, $\mathrm{RT}=$ Radiotherapy, FU= Follow up, FAC=5 Fluorouracil, Adriamycin, Cyclophosthamide, $\mathrm{AC}=\mathrm{Adriamycin}$, Cyclophosphamide, $\mathrm{T}=$ Taxane

TABLE 7: Comparison of mean FEV1/FVC ratios (\%) at different points of time and follow up period in the anthracycline based CT+RT group

\begin{tabular}{ccc}
\hline \hline Different points of study time & \multicolumn{2}{c}{ P values of mean FEV1/FVC ratio as compared with baseline } \\
& Anthracycline based CT+RT & A+T based CT+RT \\
\hline Baseline and mid cycle chemotherapy & 0.97 & 0.89 \\
Baseline and end of chemotherapy & 0.78 & 0.72 \\
Baseline and end of radiotherapy & 0.73 & 0.69 \\
Baseline and 1 month follow up & 0.087 & 0.08 \\
Baseline and 6 months follow up & 0.02 & 0.001 \\
\hline \hline
\end{tabular}

$\mathrm{P}<0.05$ "means statistically significant"

The FEV1/FVC ratio(in percentage) also revealed a definite decreasing pattern over different treatment time in the entire study group as evidenced from Table 4 and Figure 2,which declines at statistically significant level as compared to baseline at 6th month follow up with $\mathrm{p}$ value 0.003 (Table 4). Separate analysis of mean FEV1/FVC ratios over time in anthracycline based chemotherapy and radiotherapy group (Table 5) as well as anthracycline and taxane based chemo- therapy and radiotherapy group (Table 6) showed a similar declining pattern, which are statistically significant at 6 months follow up as compared to baseline in both the groups with p values 0.02 and 0.001 respectively (Table 7). 


\section{Discussion}

Regarding pulmonary toxicities following treatment with chemotherapy and adjuvant radiotherapy in breast cancer patients several studies showed definitive influence of treatment on pulmonary function tests.

Tse-Kuan $\mathrm{Yu}$ et al. ${ }^{16}$ in a phase 3 randomized study have shown that Patients with breast cancer treated with sequential paclitaxel, FAC, and radiation therapy appeared to have a very low rate of clinically relevant radiation pneumonitis that was no different from that of patients treated with FAC alone. But there was significant decrease in DLCO (diffusion capacity of lung for carbon monoxide) at long term (1 year) follow up.

Another study ${ }^{12}$ have demonstrated that loco-regional radiotherapy in breast cancer results in reductions of DLCO, VC, FEV1 and RV. The slight reduction of FEV1 was completely explained by the decrease in $\mathrm{VC}$, as the relation between FEV1 and VC was unchanged. Thus, no sign of obstructive disease was found. We suggest that the reduction of VC reflects decreased parenchymal elasticity in the irradiated part of the lung. The somewhat larger decrease in DLCO may also indicate an inflammatory reaction in the interstitial tissues.

In 1992, Marks et al. ${ }^{17}$ reported severe pulmonary complications in $10 \%$ of the patients receiving loco-regional radiotherapy following high-dose chemotherapy (including carmustine) resulting in premature discontinuation of their radiotherapy. Dose-intensified chemotherapy (FEC-based) and loco-regional radiotherapy were not associated with increased pulmonary toxicity in our material and no course of radiotherapy treatment was prematurely discontinued. Gage et al. ${ }^{18}$ have reported similar results to ours using the CTC high-dose chemotherapy. Thus, the results of Marks et al. ${ }^{17}$ may have been influenced by delayed carmustine-induced pulmonary toxicity.

In our study, the breast cancer patients received either 6 cycles of FAC chemotherapy followed by adjuvant radiotherapy or 4 cycles of AC followed by taxane and adjuvant radiotherapy. Among total 58 study population only 2 patients developed overt radiological feature of radiation pneumonitis at 6 months follow up, definitive deterioration of pulmonary function tests were observed. Both the FVC (Forced vital capacity) and FEV1/FVC ratio have shown definitive declining trend with chemotherapy and radiotherapy and mean of those decreased to statistically significant level at $6^{\text {th }}$ month of follow up. FEV1/FVC ratio decreased below $70 \%$ in about $67 \%$ patients at 6 months follow up. Separate analysis of PFTs in two different chemotherapy groups has shown similar changes.

Breast cancer treated with taxane and anthracycline-based chemotherapy regimens followed by radiation though did not develop overt radiation pneumonitis at significant levels, but declining trends of PFTs warn to continuously monitor those at different treatment times and follow ups. Before starting chemotherapy and radiation the baseline PFT must be within normal limit. Because both of these treatments have the potential to increase survival in properly selected patients, our data provide evidence that both treatments can be given sequentially without a concern for potential interactions that could result in a very serious pulmonary complication, but they result in definitive pulmonary damage.

\section{Conclusion}

The conclusion of our study was following anthracycline and/ taxane based chemotherapy and radiotherapy the breast cancer patients have shown definitive decrease in forced vital capacities and FEV1/FVC ratios. The declines were statistically significant at $6^{\text {th }}$ month of follow up.FEV1/FVC ratio decreased below $70 \%$ in about $67 \%$ of patients at 6 months of follow up.

\section{Conflict of interest}

The authors declare that they have no conflicts of interest. The authors alone are responsible for the content and writing of the paper.

\section{References}

1. Parkin DM, Whelan SL, Ferlay J, Raymond L, Young J.(eds) Cancer incidence in five continents VII. International agency for research on cancer, Lyon, France. IARC Sci Pub No. 143; 1997.

2. National Cancer Registry Programme Biennial Report (1988-89): An epidemiological study. Indian Council of Medical Research, New Delhi; 1992.

3. Berry DA, Cronin KA, Plevritis SK, et al. Effect of screening and adjuvant therapy on mortality from breast cancer. N Engl JMed 2005; 353:1784.

4. Nabholtz JM, Gligorov J. The role of taxanes in the treatment of breast cancer. Expert Opin Pharmacother 2005; 6:1073-94.

5. Shannon VR, Price KJ. Pulmonary complications of cancer therapy. Anesth Clin N Amer 1998; 16:563- 86 .

6. Ramanathan RK, Reddy VV, Holbert JM, et al. Pulmonary infiltrates following administration of paclitaxel. Chest 1996; 110:289-92.

7. Dimopoulou I, Galani G, Dafni U, et al. A prospective study of pulmonary function in patients treated with paclitaxel and carboplatin. Cancer 2002; 94:452-8. 
8. Henderson IC, Berry DA, Demetri GD, Cirrincione CT, Goldstein LJ,Martino S, et al. Improved outcomes from adding sequential paclitaxel but not from escalating doxorubicin dose in an adjuvant chemotherapy regimenfor patients with node-positive primary breast cancer. J Clin Oncol 2003; 21:976-83.

9. Mamounas EP, Bryant J, Lembersky BC, Fisher B, Atkins JN, Fehrenbacher L, et al. Paclitaxel (T) following doxorubicin/cyclophosphamide (AC) as adjunct chemotherapy for node-positive breast cancer: results from NSABP B-28 [abstract 12]. Proc ASCO 2003; 22:4.

10. Overgaard M, Hansen PS, Overgaard J, Rose C, Andersson M, Bach F, et al. Postoperative radiotherapy in high-risk premenopausal women with breast cancer who receive adjuvant chemotherapy. Danish Breast Cancer Cooperative Group 82b Trial. N Engl J Med 1997; 337:949 -55.

11. Early Breast Cancer Trialists' Collaborative Group. Favourable and unfavourable effects on long-term survival of radiotherapy for early breast cancer: an overview of the randomised trials. Lancet 2000; 355:1757-70.

12. Lind PA, Marks LB, Hardenbergh PH, Clough R, Fan M, Hollis D, et al. Technical factors associated with radiation pneumonitis after local /regional radiation therapy for breast cancer. Int J Radiat Oncol Biol Phys 2002; 52:137- 43.

13. Botterman J, Tasson J, Schelstraete K, Pauw els R, VanDerStraeten M, DeSchryver A. Scintigraphic, spirometric and roentgenologic effects of radiotherapy on normal lung tissue. Chest 1990; 97:97-102.

14. Kaufman J, Gunn W, Hartz AJ, et al. The pathophysiologic and roentgenologic effects of chest irradiation in breast carcinoma. Int J Radiat Oncol Biol Phys 1986; 12:887-893.

15. Quanjer PH. Standardized lung function testing. Report from working party 'Standardization of lung function tests', European Community for Coal and Steel. Bull Eur Physiopathol Resp 1983; 19:1-27.

16. Yu TK, Whitman GJ, Thames HD, Buzdar AU, Strom EA, Perkins GH, Schechter NR, McNeese MD, Kau SW, Thomas ES, Hortobagyi GN, Buchholz TA. Clinically relevant pneumonitis after sequential paclitaxel-based chemotherapy and radiotherapy in breast cancer patients. J Natl Cancer Inst. 2004 17; 96:1676-81.

17. Marks LB, Halperin EC, Prosnitz LR, et al. Post-mastectomy radiotherapy following adjuvant chemotherapy and autologous bone marrow transplantation for breast cancer patients with $\geqslant 10$ positive axillary lymph nodes. Int J Radiat Oncol Biol Phys 1992; 23:1021-1026.

18. Gage I, Bond S, Davidson NE, et al. Minimal acute toxicity with locoregional radiation therapy follow ing 2 high-dose chemotherapy regimens (HDC) supported with autologous marrow/stem-cell transplantation in high risk breast cancer patients (abstract). Prog/ProcASCO1998; 17:120a. 